\title{
Erratum to: Revisión sistemática de estudios de calidad de vida y/o productividad laboral en pacientes tratados con natalizumab
}

\author{
María Echave $^{1} \cdot$ Itziar Oyagüez $^{1} \cdot$ Virginia Casado Ruiz $^{2} \cdot$ Ricardo Ginestal $^{3} \cdot$ Miguel Ángel Casado $^{1}$
}

Published online: 15 February 2018

(c) Springer International Publishing AG, part of Springer Nature 2018

Erratum to: PharmacoEcon Span Res Artic (2018) https://doi.org/10.1007/s40277-017-0074-1

En el presente artículo se han detectado las siguientes erratas en los párrafos que se indican a continuación:

Resultados: (...) Dos artículos adicionales eran análisis conjuntos de los estudios AFFIRM y SENTINEL, y los tres restantes eran análisis post-hoc del estudio AFFIRM, ambos estudios de fase III de natalizumab.

La información correcta en negrita: Dos artículos adicionales eran análisis conjuntos de los estudios AFFIRM y SENTINEL, y los dos restantes eran análisis post-hoc del estudio AFFIRM, ambos estudios de fase III de natalizumab.

Results: (...) Two remaining were metaanalysis of the AFFIRM and SENTINEL studies, and three additional ones were provided by post-hoc analysis of the AFFIRM trial, phase III studies of natalizumab.

Información correcta en negrita: (...) Two remaining were metaanalysis of the AFFIRM and SENTINEL studies, and two additional ones were provided by post-hoc analysis of the AFFIRM trial, phase III studies of natalizumab.

Sección Estudios incluidos: (........ y en tres se aportaban post-hoc análisis del ensayo AFFIRM que relacionaban la capacidad visual [31] y la velocidad de desplazamiento $[23,32]$ con la calidad de vida.

The online version of the original article can be found under https://doi.org/10.1007/s40277-017-0074-1.

$凶$ M. Echave

mechave@porib.com

1 Pharmacoeconomics \& Outcomes Research Iberia (PORIB), Madrid, España

2 Servicio de Neurología, Hospital de Mataró, Mataró, Barcelona, España

3 Servicio de Neurología, Fundación Jiménez Díaz, Madrid, España
Información correcta en negrita: $(. . . . . . . y$ en tres se aportaban post-hoc análisis del ensayo AFFIRM que relacionaban la capacidad visual [31] y la velocidad de desplazamiento $[\mathbf{2 5}, 32]$ con la calidad de vida.

Sección Estudios de calidad de vida relacionada con la salud en la práctica clínica habitual y ensayos de medidas de salud percibidas por los pacientes

En el ensayo TIMER [23] se observaron incrementos significativos $(p<0,0001)$ en la velocidad de desplazamiento en la prueba de $100 \mathrm{~m}$ (timed 100-m walk) a las 24 y 48 semanas del tratamiento con natalizumab comparado con el valor en el estado basal.

Información correcta en negrita: En el ensayo TIMER [25] se observaron incrementos significativos $(p<0,0001)$ en la velocidad de desplazamiento en la prueba de $100 \mathrm{~m}$ (timed 100-m walk) a las 24 y 48 semanas del tratamiento con natalizumab comparado con el valor en el estado basal.

Sección Análisis combinados o post-hoc de estudios fase III de eficacia de natalizumab

Los análisis post-hoc del estudio AFFIRM [23] y AFFIRM junto a SENTINEL [32], que evaluaron la deambulación en los pacientes tratados con natalizumab, detectaron un incremento de la proporción de pacientes con mejoras de la velocidad en el test T25FW del 78\% (12,3\% frente a $6,9 \% ; p=0,00133$ ) en comparación con placebo tras dos años de tratamiento [23], ...

Información correcta en negrita: Los análisis post-hoc del estudio AFFIRM [25] y AFFIRM junto a SENTINEL [32], que evaluaron la deambulación en los pacientes tratados con natalizumab, detectaron un incremento de la proporción de pacientes con mejoras de la velocidad en el test T25FW del 78\% (12,3\% frente a $6,9 \% ; p=\mathbf{0 , 0 1 3 3})$ en comparación con placebo tras dos años de tratamiento $[25] \ldots$ 
FIGURA 1.

En la figura 1 se han detectado los siguientes errores, incluyendo la información correcta en negrita:

Referencias tras eliminación de duplicados: $n=84$. Información correcta $\boldsymbol{n}=\mathbf{8 0}$

Trabajos revisados: $n=84$.

Información correcta $\boldsymbol{n}=\mathbf{8 0}$
Estudios de práctica habitual $n=4$. Información correcta $\boldsymbol{n}=\mathbf{6}$

Ensayos sobre $\mathrm{PRO} n=4$. Información correcta $\boldsymbol{n}=\mathbf{3}$

Post-hoc análisis AFFIRM $n=3$. Información correcta $\boldsymbol{n}=\mathbf{2}$ 